\title{
Creating a Sustainable Partnership Between a Science Center, University, and Local School Districts: A Retrospective on Over 20 Years of Successful Programming and Partnership
}

\author{
Katie Busch Chandran ${ }^{1}$, Kevin Jarrett', and James Michael Wyss ${ }^{1,2}$ \\ ${ }^{1}$ Center for Community Outreach Development, College of Arts and Sciences and ${ }^{2}$ Departments of Cell Developmental and Integrative Biology, Medicine and Biology, \\ University of Alabama at Birmingham, Birmingham, $A L$ \\ Keywords: STEM, Museum, Science Center, University Partnership, Science Outreach, Biotechnology, High School, Middle School, Sustained Partnership, Inquiry \\ Publication Date: October 21, 2020 \\ DOI: https://doi.org/10.15695/jstem/v3i3.03
}

\begin{abstract}
The University of Alabama at Birmingham (UAB) and the McWane Science Center have partnered for over 20 years to provide secondary students in Alabama opportunities to conduct inquiry-based, standards-aligned science labs. LabWorks (middle school) and GENEius (high school) programs offer multiple research laboratory experiences in which students explore molecular biology, physical science, genetics, engineering, anatomy, and forensic science, with associated summer professional development for teachers: BioTeach and GeoTeach. These programs each attract 3,000-4,000 participants annually. This partnership has been synergistic, allowing the science center to offer high-level secondary science programming, while giving the university a conduit into the K-12 world. Originally, these programs were developed through Howard Hughes Medical Institute funding, and then expanded through support from the National Institutes of Health, the National Science Foundation, and the US Department of Education. These programs have excited secondary students and teachers about science education and careers, provided authentic science experiences, and given teachers the opportunity to experience a model of learning that engages students in "real science" closely linked to their curriculum. For over 20 years, this unique, evolving partnership has increased the understanding of over 90,000 students and teachers relative to the opportunities that science and STEM careers hold.
\end{abstract}

\section{INTRODUCTION}

Universities are hubs of knowledge and understanding that are especially important in the rapidly changing science, technology, engineering and math (STEM) disciplines. While researchers are outstanding in their ability to deeply probe critical science questions and pass these abilities on to their trainees and students, they are much less adept at sharing their skills, research results, and understanding of societal implications with the world outside of the research community (Ecklund et al., 2012; Farah, 2019). Almost 30 years ago, a team of University of Alabama at Birmingham (UAB) faculty researchers from multiple schools partnered with science educators in area K-12 schools with a goal of improving the readiness of students for STEM-related careers and college programs. This resulted in a continuously evolving program to provide K-12 students and their teachers with understanding and excitement about STEM and the opportunities it holds for them and the future.

Realizing the importance of diversity among personnel in biomedical research and health care in the state and nation, in 1998 the President of UAB in partnership with the Dean of Medicine created the Center for Community Outreach Development (CORD) as a mechanism to advance STEM learning. Although CORD's doors would not be closed to any students, the goal was to particularly target working with underserved schools, so that the students would be able to access a pathway to STEM careers, including clinical health careers. In 2000 CORD was expanded and placed under the leadership of the UAB Provost who actively supported the mission of STEM outreach. In 2007, the program oversight was transitioned to the School of Education and the College of Arts and Sciences at UAB to foster partnerships in outreach among CORD, the UAB faculty and UAB students, who will excite and teach the next generation of STEM professionals. Today, the Center has a dual reporting line to the UAB Provost and the Dean of the College of Arts and Sciences, and it operates on a budget of about $\$ 4,000,000$ per year; about $97 \%$ of which is extramurally funded. Since its inception. CORD has worked to bring together faculty members from the medical center schools, the School of Education, and the College of Arts and Sciences 
to share the goal of investing university resources into the local community. This mission of engaging and supporting the community is one often attempted by universities, but it can be difficult to achieve (Weerts, 2019). The earliest incarnation of these goals was a teacher-training program to familiarize teachers with modern techniques in biotechnology and give them access to equipment and facilities to carry out research. Typically, such facilities are only available to those at a post-secondary level. Further, students were given access to the same equipment to perform high-level, hands-on science in their classrooms. Initially, these efforts focused on education in health-related topics affecting their local communities and provided the students with the knowledge and pedagogical skills to educate their families, lead healthier lives, and encourage them to seek medical care for conditions that might have previously gone unnoticed or ignored. A major part of this effort was the creation of the GENEius lab at the McWane Science Center and CORD's BioTeach high school teacher professional development program that supports GENEius. Additionally, the Hands-On school outreach program and the high school Summer Science Institute developed out of this initiative. By 2004, the high school programs were well established and effective, enabling CORD to focus on expanding its STEM education program to the middle school level. This led to the development of a new middle school science lab at McWane Science Center (LabWorks), summer science camps for 3rd-8th grade students, and middle school programs in area classrooms. The success of the BioTeach teacher professional development program, coupled with requests from middle-school teachers to join in the training, directly led to creating GeoTeach in 2015. GeoTeach uses the same model that made BioTeach a success but focuses on building science skills for students in grades 5-8.

CORD programs bring together top researchers, field experts, undergraduate and graduate students, and postdoctoral fellows to assist in its student and teacher programs. Further, specialists with science and education backgrounds direct the K-12 programs to help bridge different academic spheres. The programs are directed by a University-based Director and Principle Investigator, who is an active and well-funded academic and researcher and is well versed in the administrative aspects of the University. We believe this leadership has played a critical role in the longevity of CORD, in CORD's ability to recruit faculty and trainees to assist in its programs, and to obtain extramural funding to sustain the program (Groark and McCall, 2018). In addition, many university and outside partners have added greatly to the projects and brought a unique depth to many of the programs. Some of the most important resources in this regard are university researchers, who share their research with teachers in workshops and students who facilitate programs and come from all UAB schools and colleges to volunteer to assist with special student programming, and countless post-docs, students, teachers, and school administrators, who have helped to develop and shape the programs through their formative feedback. CORD strives to ensure that each partnering group derives value for themselves out of their experience with the programs (e.g., medical students learn how to explain biomedical conditions to 5th graders, which in turn provides experience in communication for their medical career).

This paper provides a longitudinal retrospective of CORD's history of developing GENEius, LabWorks, BioTeach, and GeoTeach to educate, inspire and empower teachers and students in STEM education and careers, through a sustainable partnership that continues to grow and provide opportunities for students to excel in science. Each of these programs continuously evolves in response to participant feedback, new STEM education standards, changing administrations and policies, new STEM discoveries, and the perceived needs in the community. Throughout, CORD has stayed focused on the core mission of elevating the excellence of STEM education in all schools (particularly in underserved schools) and thereby increasing diversity in STEM careers (Valantine, et al., 2015). This paper also shares lessons learned and overviews of some of the partnership's most successful modules.

Program Origins. In 1992, UAB STEM faculty and secondary science teachers and administrators from 22 area school districts partnered in developing BioTeach. The goal was to provide teachers with state-of-the-art knowledge and skills to meet the increasing need for high school teachers to understand and be able to convey modern molecular biology and biotechnology and to teach the associated laboratory skills to students. The goal has been to assist teachers in gaining knowledge and skills needed to translate important areas of modern science into student knowledge and skills that increase college and career interest and readiness in STEM disciplines. BioTeach has been a major professional development course for Alabama high school biology teachers, and in several major districts graduation from BioTeach leads to "Master Teacher" status for the graduates.

During its initial years (1992-2001), BioTeach was largely paid for by university funds from the offices of the Provost and Dean of Medicine. In 2002, CORD received funding from the Alabama Commission on Higher Education (pass through from the federal "No Child Left Behind" program). That funding ended in 2016, and BioTeach has since been dependent largely on funding from its National Institute of General Medicine Science Education Partnership Awards (SEPA) and from the US Department of Education's Gaining Early Awareness and Readiness for Undergraduate Programs (GEAR UP). The three-week course offers state-of-the-art, inquiry-based experiences that have assisted 


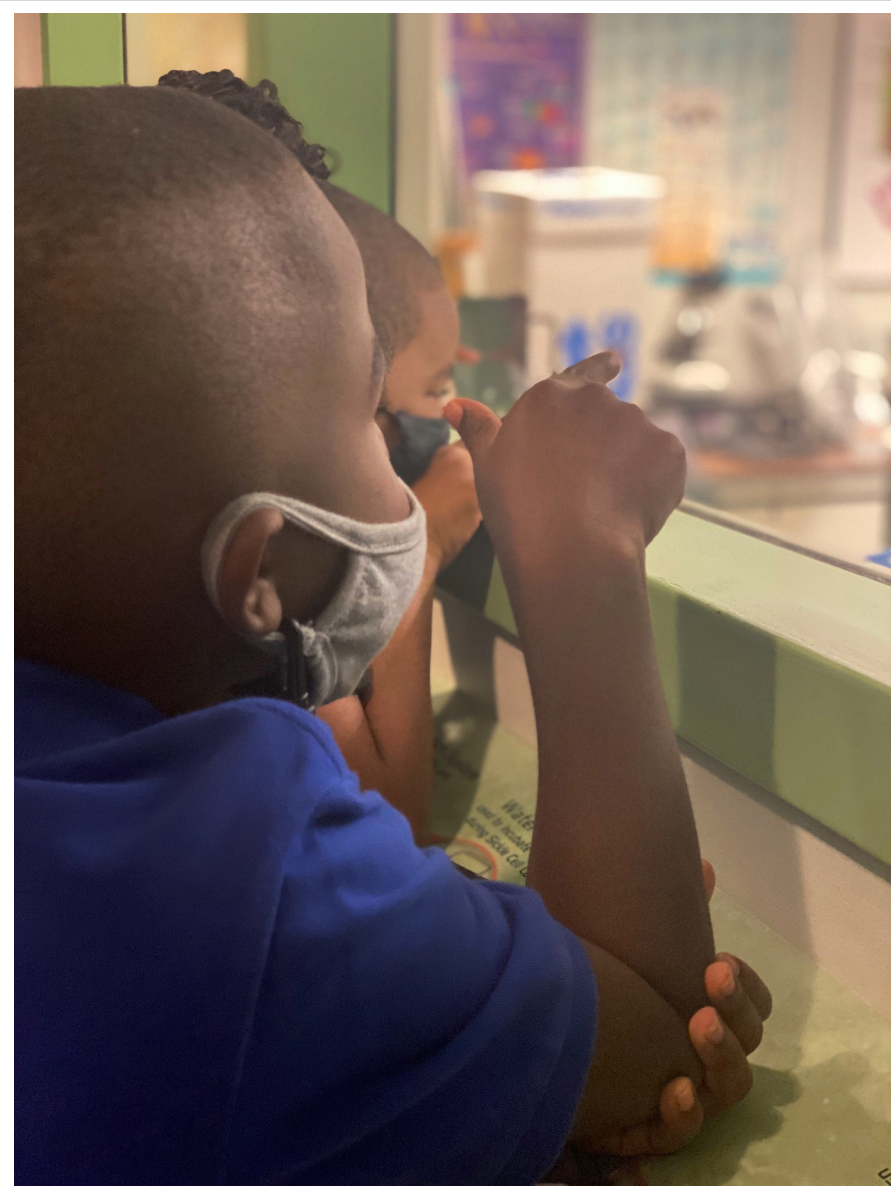

Figure 1. Two 8-year old children gaze with wonder into the science that high school students are unfolding in the GENEius Lab.

teachers in understanding science principles and how to convey these principles to their students. The teachers are also provided with loaner kits that include materials and equipment required to replicate the CORD research modules in their own classrooms.

Within the first few years of the pilot program, it became apparent that although the BioTeach training and modules increased early access to biotechnology for those who participated in the program, most teachers in the state did not have labs that could provide the students with opportunities to use modern biomedical tools. The concept of GENEius arose as a venue in which multiple classes could come to learn handson lessons in science. Since the origin of GENEius, teachers trained in BioTeach have been invited to bring their students to take part in the GENEius Lab, which offers at least four research-based laboratory experiences that are independent of the BioTeach modules. While the GENEius program was originally open to only BioTeach graduates and their classes, it is now available to all high school teachers who wish to participate. This change was needed because of the increasing demand for teachers to complete school district and state required summer training, often making them unavailable to participate in the full three-week BioTeach training. We also recognized that teachers who lived over 50 miles from

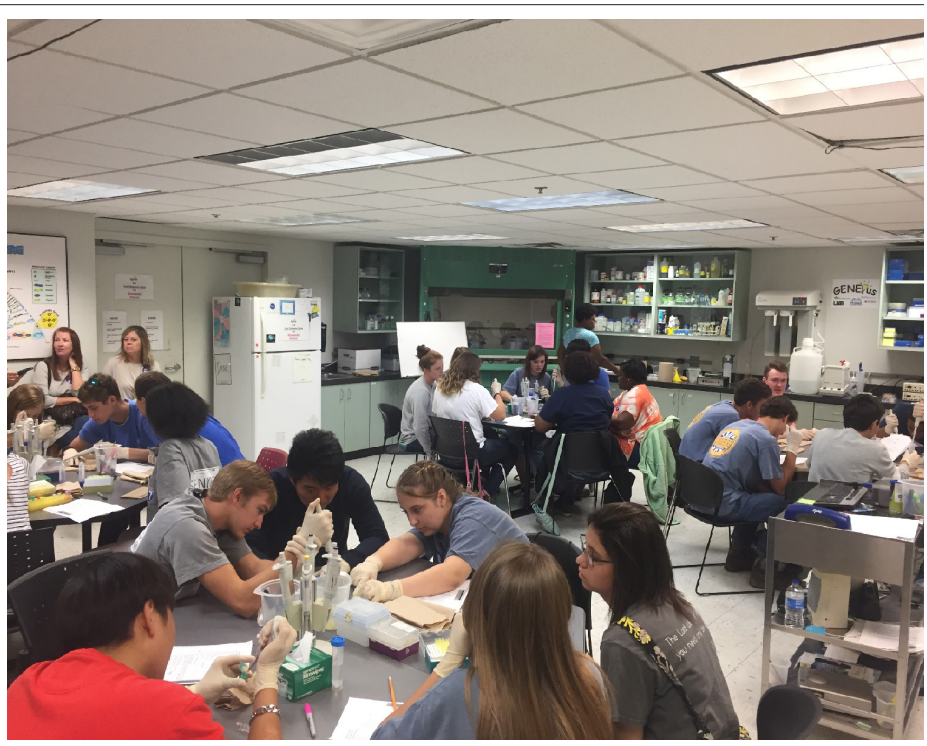

Figure 2. This figure depicts high school students participating in the GENEius laboratory. This is about the largest class we can host at one time in a single lab $(\sim 30)$.

Birmingham could not easily attend three full weeks of professional development, but they could bring their students to GENEius. This change has helped to engage more teachers and students and thereby grew GENEius for over 20 years.

Both the BioTeach training and the GENEius labs are housed at the McWane Science Center. The construction was originally funded through a gift from the Altec-Styslinger Foundation and partnership funding from UAB and McWane Science Center. As the program was opened to more participants, the nature of the partnership changed slightly, with McWane adding the GENEius labs to its program offerings and charging museum admission to the students. McWane has been committed to raising and dedicating funds from grants and local corporations to enable the cost of admission to be covered for groups that have a financial need or are designated Title I schools. CORD and McWane have continued to partner for over 20 years to use museum private and grant funds to fund the GENEius Lab. In this way the program has been sustainable by generating needed revenue, while sustaining the mission of being accessible to all teachers and students.

Since the inception of GENEius, McWane Science Center has provided UAB CORD with a dedicated GENEius lab space $(\sim 1,000$ gross square feet) on its main level. The lab space encourages museum attendees to gaze into the lab and appreciate modern science. Younger visitors are excited to use a raised step for viewing into the lab through large windows and full-length glass doors, which provide an accessible view point for all visitors. This "fish bowl" effect has a threefold positive impact (see Figures 1 and 2). First, younger children who come to the museum can watch older students working as scientists, and thus the younger students have their imaginations and excitement ignited. Recently, 
McWane added a lab and engineering studio across from the GENEius lab to channel the young-learner excitement into early experiences with STEM. Second, the high school students in the lab (and teachers in the summer) see others peeking in, often waving or knocking on the glass, and thus they feel a sense of excitement and importance about their work in the lab. Third, it provides McWane visitors with the feeling that real science goes on here. There are many museums that display "real scientists at work," but it is rare to offer a daily lab experience in which students are invited in and viewed as the "real" scientists.

In 2002, CORD expanded its partnership with McWane Science Center's middle school "Explore!" Lab, with the mission of enhancing this labs' offering for children and teachers. CORD and McWane staff recognized that middle school children have a high degree of interest in inquiry-based science experiences and are eager to engage in hands-on science opportunities of which very few were provided to them in their classrooms, especially in the underserved schools in Alabama. It was recognized that if we wait until high school to engage students, many will have already become disinterested in STEM fields. In addition, the partnership believed bringing the students to a "real" laboratory would engage them in understanding that they too could be scientists and clinical professionals. The Explore! Lab offered an informal "drop in" learning center for children and their parents; however, the more informal format of a public "drop in" program did not provide the level of rigor and understanding that the program aimed to achieve. Thus, in 2006, CORD developed LabWorks as the successor of the Explore! Lab. LabWorks was developed using the model of GENEius, with the modification of shorted two-hour sessions to accommodate the differences in students' attention span (Vawter, 2010).

Offering" half-day" sessions has enabled LabWorks to serve more students in a single school day, which is almost a necessity when working with underserved middle schools, due to their cost for substitute teachers and transportation. Many schools have 100-400 students in each grade level and are not willing or able to allow many field trips. The LabWorks and GENEius staff are all cross-trained on labs, thus providing a workforce that can offer concurrent sessions, allowing over 110 students per day in LabWorks and 60 per day in GENEius. All CORD experiences have always been aligned to the specific grades' curriculum standards.

\section{METHODS}

Program Components. Students participating in the GENEius and LabWorks programs begin the lab with an audience response system (clicker) pretest, designed to assess students' prior knowledge of an area and attitudes toward it. After the pre-assessment, the GENEius or LabWorks program facilitators present a 20-30-minute PowerPoint inter-
Table 1. GENEius Program Alignment to Next Generation Science Standards

\begin{tabular}{ll}
\hline Program & Standard \\
\hline Huntington's Disease: & HS-LS1-1, HS-LS1-2, HS-LS3-1, \\
The Trembling Brain & HS-LS3-2; AP Biology Big Idea 3 \\
HIV: Lifecycle of a Virus & HS-LS1-1, HS-LS1-2; HS-LS3-2; \\
& AP Biology Big Ideas 3, 4 \\
Sickle Cell Anemia: & HS-LS1-1, HS-LS3-1, HS-LS3-2, \\
Tracking Down a Mutation & HS-LS3-3, HS-LS4-3, HS-LS4-4; \\
& Biology Big Ideas 1, 3 \\
DNA: A Person's Ultimate & HS-LS1-1, HS-LS3-1, HS-LS3-2; \\
Fingerprint & AP Biology Big Ideas 1, 3 \\
\hline
\end{tabular}

active introduction and discussion. The introduction also includes a description of the laboratory techniques that the students will use in the laboratory, and descriptions/representative examples of possible results and an interpretation and analysis of the results. Students are then broken up into groups (maximum of 6 students) and assigned a facilitator (an undergraduate, graduate, or postdoctoral trainee), who guides the students through the protocol, assists with laboratory techniques and explains the scientific concepts throughout each laboratory experience. After the completion of the laboratory procedure students complete a posttest to evaluate their understanding and knowledge gained from the laboratory experience. Students respond to basic questions regarding the experiment and the techniques used. After the posttest, students engage in a competitive and interactive "review game" in which questions are asked about all aspects of the experience.

GENEius Laboratory Description. The GENEius program offers multiple research laboratory experiences for high school students to explore molecular biology and genetics. The availability of innovative technology at the GENEius laboratory allows the teachers to challenge their students with very complex hands-on experiments that are not typically encountered until 200 or 300-level college biology courses. A number of virtual laboratory simulators are now available online and a number of studies have found them to be effective in enhancing student understanding of class content (Tuysuz, 2010; de Vries and May, 2019). It is less clear if these virtual-only courses are as effective as hands-on experiences, since the two have not been directly compared; however, most of the literature suggests that virtual experiences are not as effective as true hands-on lab experiences, especially in the initial engagement of the students' STEM career interests and skills development (Tuysuz, 2010; de Vries and May, 2019).

The GENEius lab currently offers four research experiences for students, including exploration of sickle cell anemia, DNA fingerprinting, Huntington's Disease, and Human Immunodeficiency Virus (HIV). Each year, these programs are continuously reviewed by the CORD staff, UAB re- 
searchers, and school teachers to ensure that each one is up-to-date, elicits the interest of the students, and provides the students with an understanding of concepts covered in the science standards at their grade level (see Table 1 for Next Generation Science Standard and AP Biology Big Idea alignment; https://www.nextgenscience.org/). Further, GENEius has couched its biotechnology education in the context of socio-scientific issues such as genetic testing and gene editing, thus making GENEius more engaging and motivating for students (Chung et al., 2016; Nordqvist and Aronsson, 2019). Further, GENEius attempts to ensure that the topics covered are especially engaging to the students who are from minority populations that are underrepresented in STEM careers.

One of the original laboratory experiences at GENEius "Sickle Cell Anemia: Tracking Down a Mutation" is a fullday (9 am- $2 \mathrm{pm}$ ), inquiry-based, biology experience for high school students enrolled in genetics or advanced biology courses (Jarrett et al., 2016). In the lab, students employ restriction endonuclease digestion, cellulose acetate gel electrophoresis, and microscopy to discover which of three putative patients have the sickle cell genotype/phenotype, using DNA and blood samples from wild type and transgenic mice that carry a sickle cell mutation. The inquiry-based, problem-solving approach facilitates the students' understanding of the basic concepts of genetics, cellular and molecular biology and provides experience with contemporary tools of biotechnology. This lab is particularly interesting to African-American students, as many of them know someone who either has the disease or is a carrier for it. The lab helps them understand the causes and consequences of a genetic disease that is relatively common (1:365-1:600) in individuals of African descent in the USA (Hassell et al., 2010). The experience also increases their understanding of first principles of genetics including the Central Dogma of molecular genetics and Mendelian inheritance.

A second experience in GENEius is "DNA: A Person's Ultimate Fingerprint," in which students focus on genetic diversity and use contemporary techniques of molecular biology to isolate DNA from their cheek cells, use polymerase chain reaction (PCR) to amplify a highly variable region of chromosome 1, and employ gel electrophoresis to analyze DNA samples of the D1S80 gene (a non-coding region of polymorphic DNA; D1S80 is similar to VNTR used in forensic analysis, but is rarely used in a professional case/profile). Students quantify their inherited copies of D1S80 by approximately measuring their sequence against a 100-1000 $\mathrm{kb}$ DNA ladder. It is an exciting experience for them to examine their own DNA and connect principals of genetics to forensic science.

The third GENEius offering is "HIV: Lifecycle of a Virus," in which students explore the lifecycle of HIV and test for the presence of the antigen by utilizing an Enzyme
Linked Immunosorbent Assay (ELISA). Public health issues relating to AIDS epidemiology and HIV transmission are also addressed through class discussion and a mock fluid exchange. While this program was very popular with students 10 years ago, most students now believe that HIV/AIDS is cured, so they are less interested. This is an example of the type program that we critically consider for replacement with one that is of more current interest.

The final day long GENEius experience is "Huntington's Disease: The Trembling Brain," which focuses on both normal and diseased states of the brain. In it students dissect a sheep brain and trace neuronal sensory pathways. Students also use agarose gels and semi-log graphs to identify pathological DNA samples of a mock family, and they use paper gene models to demonstrate their understanding of the defect in this genetic disease. Ethical issues surrounding genetic testing and the potential for gene editing with CRISPR-Cas9 are discussed by beginning with the question: "Would you want to know if you had a genetic disease?"

BioTeach Program Description. BioTeach is an intensive three-week course in molecular biology for small groups of high school teachers (15-20). BioTeach participants have the option of earning graduate credit hours and/or professional learning credit (for teaching certificate renewal) upon completion of the program. When BioTeach began, there was a great need for high-level, integrated science training for teachers, especially to allow teachers to gain skills in technologies that were quickly emerging in biomedical sciences. That need continues as science rapidly progresses. BioTeach training has continuously evolved to provide the latest science knowledge and skills to the participating teachers (Jacobi et al., 2019). The first week of BioTeach training is devoted to learning basic biotechnology skills (e.g. measurements, pipetting, and bacterial culture). The second and third weeks allow teachers to become proficient in sophisticated techniques such as gene cloning, DNA isolation, DNA and protein gel electrophoresis, PCR, and restriction endonuclease analysis. Teachers are also trained in facilitating inquiry-based learning (vs. leading students in labs). This is a critical component in helping students develop the ability to conduct authentic research (Bielik and Yarden, 2016). BioTeach integrates GENEius laboratories, BioTeach modules, and state-provided Alabama Science in Motion (ASIM) modules to provide excellent training for the BioTeach graduates, so that they can utilize all of the resources that CORD makes available to them and their students during the academic year. To date, over 450 teachers have graduated from BioTeach.

In addition to the in-house laboratory experience, BioTeach also offers four ready-for-classroom modules, which are designed for several days of instruction in the teachers' classrooms. Modules are loaned to teachers with 
all of the equipment, supplies, and reagents necessary to perform inquiry-based experiments in microbiology and molecular biology. This provides access to state-of-the-art equipment and experiments that are not available in many of the underserved schools. Throughout the workshop, teachers work in teams and switch between roles of learners training on the modules and implementers considering how they would optimize the modules for their classrooms. This allows them to be immersed in inquiry-based pedagogy, experiencing making discoveries on their own, and then turning it around and planning deliver of the experience to students. The BioTeach loaner modules include:

1. In "Measurement and Growth: An Introduction to Molecular Techniques,"students learn how to use modern equipment, grow E. coli on plates and in liquid media, use the scientific method, and design experiments. Specifically, they learn how to streak for single bacterial colonies on agar plates. They then measure the growth rates of different strains of bacteria under various environmental conditions. The students also swab their environment for microorganisms and design experiments to study the effect of "nonstandard" conditions on bacterial growth.

2. "Ultraviolet Mutagenesis" allows students to generate ultraviolet (UV) dose response curves for two strains of $E$. coli. They also examine the effectiveness of various sun blocking agents. Students then design their own experiments using UV-sensitive and UV-resistant bacteria, a UV light source, and various UV blocking agents. This module has enabled teachers to effectively introduce the scientific method and experimental design in their classrooms.

3. "DNA-Mediated Transformation of Bacteria" helps students understand that DNA is the genetic material for all organisms. By treating E. coli with a cold calcium chloride solution and exposing E. coli to a plasmid containing pGLO (a gene for green fluorescent protein), students can transform the genome of $E$. coli. Next, they selectively grow cells on plates containing ampicillin and arabinose and the successful transformation is noted

Table 2. LabWorks Program Alignment to Next Generation Science Standards

\begin{tabular}{ll}
\hline Program & Standard \\
\hline Toothpaste Chemical Engineering & MS-PS1-2, MS-PS1-3, MS-PS1-5 \\
A Light in a Dark Room & MS-PS1-1, MS-PS1-2, \\
& MS-PS4-1, MS-PS4-2 \\
The Eyes Have It & MS-LS1-2, MS-LS1-4, \\
& MS-LS2-1, MS-LS2-2 \\
Isn't That a Crime & MS-LS3-1, MS-LS3-2 \\
Environmental Mystery & MS-ESS2-4, MS-ESS3-3, \\
& MS-ESS3-4 \\
\hline
\end{tabular}

with the use of UV light irradiated plates. This module reinforces the fundamentals of experimental design.

4. "Detection of Genetically Modified Crops" module was developed due to the increase interest in, and popularity of, genetically modified crops. Using DNA amplification techniques (PCR) from student-chosen foods followed by DNA analysis (gel electrophoresis), students test for the presence of multiple DNA sequences associated with genetically modified organisms. Students will then make the diagnostic determination of the extent to which their foods are genetically modified.

About $75 \%$ of BioTeach graduates annually bring their classes to the GENEius laboratory and request BioTeach Modules for their classrooms. GENEius experiences are fun for the students and good learning encounters, giving students a taste for biotechnology work and applications. That experience can be enhanced by their teachers' active participation in the GENEius sessions and can be extended into the classroom by teachers who are trained to use the BioTeach loaner modules. This gives the teachers ownership in the education process and encourages them to make full use of the modules, which in turn helps them to implement hands-on science in their classrooms and successfully teach higher-level content and skills (Table 2; Elton-Chalcraft et al., 2020).

LabWorks Laboratory Description. For 20 years, Alabama has followed a model of teaching earth science in 6th grade, life science in 7th grade, and physical science in 8th grade, with engineering integrated into each grade (following the implementation of the Next Generation Science Standards (NGSS; https://www.nextgenscience.org/) in 2015, NGSS). LabWorks' goal is to offer two standards-aligned modules per grade level (see Table 3 for NGSS alignment). Some modules have endured for the lifespan of the program, with continuous updates, but other modules have been retired and replaced with new modules that are better fits and more engaging for current students and NGSS.

1. In "Toothpaste Chemical Engineering," 8th graders take a mundane activity and turn it into an exciting engineering goal. They learn about the chemical composition of their teeth and the reactions that keep them strong. They use plaque-disclosing tablets to see where build up occurs and scrub it away with toothbrushes and toothpaste provided by a partnership with UAB's School of Dentistry. Then they test saliva and toothpaste $\mathrm{pH}$ and learn about the most common ingredients in toothpaste and the function of each component. After testing the effectiveness of several brand-name toothpastes relative to scrubbing away stains on an egg shell, they take on the role of chemical engineers, using household chemicals to design their own toothpaste for a specific dental pur- 
Table 3. GENEius Post-Lab Teacher Inventory

1. The structure and organization of the lab experience

was optimal.

2. The length and content of the ore-lab lecture was appropriate.

3. There was a sufficient time to complete the experiment.

4. There was adequate time to discuss and interpret results.

5. My students seemed to understand the experiment.
Scale 1-5

(1= Strong-

ly Agree,

$5=$ Strongly

Disagree)
6 . There was enough time to visit the McWane Center exhibits.

7. Overall, our GENEius lab experience was a success.

pose (e.g., tartar removal, whitening, gum disease, etc.), and then test the effectiveness of their new creations.

2. In "A Light in a Dark Room," 8th grade students explore the connection between two ends of the NGSS standards: elements and waves. Students are introduced to astrophysics by making the same discoveries that early scientists made about light, wavelength, energy, temperature, and color. They then learn how to recognize an element's signature spectra and identify mystery compounds with spectroscopes. To emphasize the relationship between light and energy, they hunt for fluorescent compounds and design their own invisible ink.

3. One of our most enduring 7th grade labs, "The Eyes Have It," facilitates student learning about the structure and function of the eye as an organ and on a cellular level. They relate this knowledge to understanding how illusions work and thinking about designing robotic sensors. After a series of engaging demonstrations and activities, they experience what is often their first dissection of an organ. Students work in groups to carefully cut into and examine the structures of a cow or sheep eye from macro structures like the iridescent tapetum lucid$u m$ to the cone and rod cells in the retina.

4. "Isn't That a Crime" is a fan-favorite lab for all middle school students, although it is best suited to accompany 7 th grade life science standards. After being introduced to the field of forensics, students are briefed in a non-frightening case and are tasked to help with the investigation. Working as "technicians in training," students conduct a simulated blood test, while learning about proteins, antibodies, the immune system, simple Mendelian genetics, and Punnett squares. Next, they learn how to use a micropipette and load an agarose gel with DNA samples. They are also taught how to read and understand a DNA fingerprint. While their electrophoresis runs, they collect their final pieces of evidence, dusting for and comparing fingerprints and examining hair and fibers under microscopes. When the gel finish- es, they compile all evidence and make a case for the suspect they think is guilty.

5. Forensics isn't all blood, guts, and human stuff. In an "Environmental Mystery," 6th graders have the opportunity to solve an earth science case. Students are introduced to a variety of ways water can be polluted through human activity and the kinds of contaminants that can lurk invisibly in the water. They are given a backstory to a fictional city with a pollution problem and a map of test sites. With this in hand, they are tasked with testing samples for microbial, plastic, metal, and other contaminants as EPA agents. Using test results and inference, students determine the source of pollution and notify "town authorities."

University Trainees' Partnership. To support authentic lab experiences, CORD has been able to recruit undergraduate and graduate STEM majors from UAB to serve as paid facilitators for GENEius and LabWorks. These students are attracted to the opportunity to work with equipment they have used or may use in future lab work, as well as being able to inspire young people to pursue STEM careers. Further, we stress to the UAB students that they will gain great communication skills: i.e., if you can teach it to a teenager, you are well on your way to communicating your research and STEM knowledge to the public and to other scientists. Staffing the GENEius and LabWorks with skilled and enthusiastic facilitators (composed of undergraduate, graduate, and post-doctoral students) and directors has been an important factor in increasing participation in each to over 3,000 student participants each year and a combined total about 100 classes. Over $90 \%$ of the teachers annually return with their classes, with many noting the knowledgeable and very engaged staff as being a valuable part of the experience.

\section{RESULTS}

Program Assessments. Teacher and student feedback aid in the process of continuous optimization of the GENEius and LabWorks experiences. The GENEius lab not only has a greater than $90 \%$ return rate, but many BioTeach graduates will take BioTeach a second time to sharpen their laboratory skills and stay apprised on the latest biotechnology trends. Typically, teachers are allowed to retake the course every five years, a period during which about $40-50 \%$ of biotechnology knowledge and techniques have evolved. The following is a statement on the impact of BioTeach from a previous graduate:

BioTeach provided the spark I needed to ignite not only renewed interest in my teaching, but it also kindled excitement for student learning. Prior to BioTeach, I had not used micropipettes or had any 


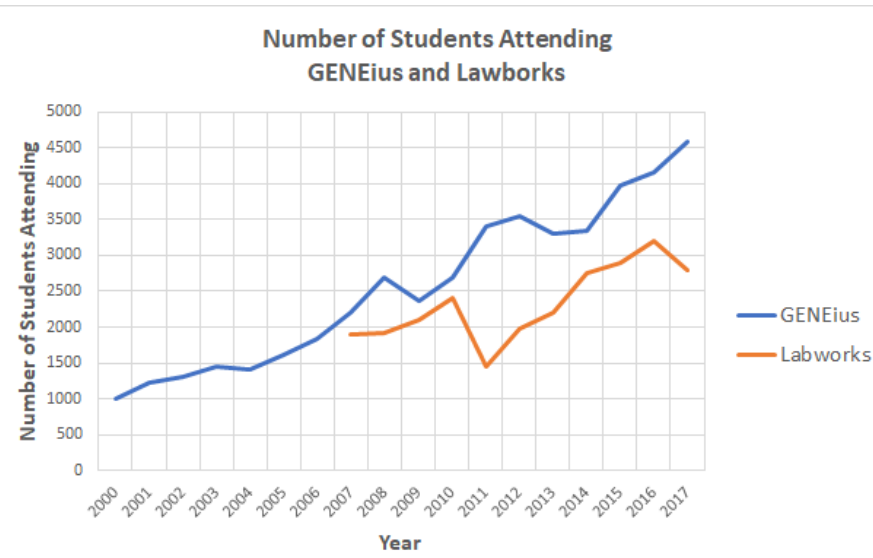

Figure 3. This figure shows the number of students who attended GENEius and LabWorks in the years listed.

experience with gel electrophoresis or PCR. I was eager to use biotechnology equipment and thrilled to add the latest biotechnology component to my classroom. I learned a great deal from the "handson" component, as well as, the lectures from visiting UAB professors. Because of BioTeach, I know I became a better teacher and my students became more competent learners. It was also a bonus knowing that the CORD staff was only a phone call away.

Throughout the existence of GENEius and LabWorks, BioTeach teachers have participated in the development and refinement of the science offerings. As their final BioTeach experience, the teachers present classroom education modules that they will use in their own classrooms. Although the partnership with other teachers who visit the GENEius lab is not a formal one, their formative feedback also has been used and valued throughout the years to improve the program. In an attitude inventory with items such as "the structure and organization of the lab experience was optimal," "the length and content of the pre-lab lecture was appropriate," and "my students seemed to understand the experiment." Approximately $70 \%$ of teachers responded with "strongly agree" to all seven items (see Table 2 for full inventory). Of the $\sim 30 \%$ who did not give all "strongly agree responses" the majority gave "strongly agree" to all items except an "agree" response to either the item "there was enough time to visit the McWane Center exhibits," or "my students seemed to understand the experiment." One hundred percent of participants gave a "strongly agree" to the item "overall, our GENEius lab experience was a success," regardless of how they rated other items.

In additional to responding to a scaled opinion inventory, teachers were asked to answer short response questions about the lab space and program. The following quotes from participants are representative of all responses given over the years:

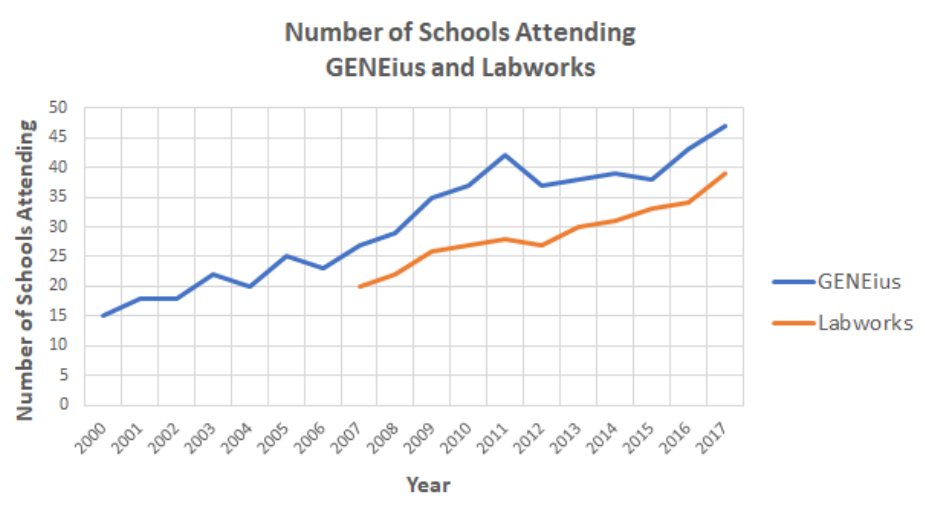

Figure 4. This figure shows the number of different schools attending GENEius and LabWorks in the years listed.

"[The GENEius lab experience is] somewhat different because we don't have nearly the resources in the classroom as we did here. That makes all the difference in the world."

"This experience was different from my classroom because the students were exposed to a real lab setting, working with graduate students [who were] knowledgeable about the content of the lab."

"This is a wonderful program. People were nice and considerate. Facilitators were very knowledgeable about the subject matter. This was the most fun and interesting time the students have ever had when it relates to science labs."

"The greatest impact this lab had on my students was being able to use the equipment to apply concepts learned in class."

"My students were really interested in sickle cell anemia. They received an excellent version of what we taught in class."

Many teachers noted that the lab was different from their classrooms because of the equipment available to students, the real lab feel (not just a set up in their classroom), the opportunity to perform "real science" and the depth of content covered. A few teachers, who reported that the lab was similar to their classrooms, still cited these factors as reasons they valued the experience. All participants who responded to the question, "Would you recommend the GENEius lab to your colleagues?" emphatically indicated that they would. Although part of feedback mechanism is collecting quantitative data, e.g., student score gains, such qualitative information collected has been equally helpful in developing high-quality programming for teachers and their students.

Since 2000, the GENEius and LabWorks programs' average annual student attendance has approximately doubled (Figure 3). The attendees include out of state participants (average approximately 200/year) traveling from Mississip- 
pi, Georgia, Tennessee, and Florida. Over $60 \%$ of all students in GENEius have been underrepresented minority students, including students from rural, underserved school districts.

The number of schools attending GENEius and LabWorks has also increased yearly (Figure 4). The GENEius lab is limited to the number of students that it can accommodate daily. To maintain a low student to teacher ratio $(6: 1)$, groups are limited to 24 students per GENEius laboratory experience per day. In 2011, additional laboratory space was opened to accommodate 54 students per day. Still, some schools with larger groups attend the GENEius lab on multiple days, to give all students the opportunity to participate in the experience. Many of the schools sending students to GENEius made multiple visits to the same lab experience to accommodate their large number of students. Further, 35\% of the schools return to participate in different GENEius lab offerings each year. By linking the experiences to the NGSS, teachers can choose a lab that best fits the STEM learning needs of their students.

Students are tested for content knowledge gains after completion of all of our programs. Across both GENEius and LabWorks, students score an average of 4/10 on the pretest and $8 / 10$ on the post-test evaluations, which probe content knowledge and ability to use that knowledge.

After GENEius labs, students are able to answer high-level content questions, recalling novel content during a competitive team game. The lab facilitator asks a question and all teams have the opportunity to discuss and write a response on a white board. All groups who answer correctly receive points. The game can be easily modified to ask a range of questions, and teachers often add incentives like a bonus point or homework credit encourage their students to give the game their best effort. Most teams score within a few points of each other, indicating that although there is a "winner," all students have acquired new knowledge and understanding during the lab. Research on a similar program has demonstrated that after a hands-on biotechnology lab experience, students show an improved attitude towards science and scientific research, independent of content knowledge gains (Jarrett et al., 2016; Yang et al., 2017).

Students in LabWorks show similar knowledge and skills gains. After LabWorks, they can add much more to concept maps that they initially drew out before the LabWorks session. They greatly increase the level of detail and understanding. When probed for their interest in STEM careers, they show greatly increased interest after the sessions, especially for those careers introduced during the lab program. They also express enthusiasm for the activities. Some groups come to the lab after being well prepared by their teacher prior to the visit. Others attend the same program with no prior content knowledge or preparation and sometimes without any hands-on lab skills or past experiences. Instructors and facilitators ask probing questions during the initial lec- ture to assess student prior knowledge and make modifications to the lesson plan in response to their assessment of the students' level of STEM understanding. This ranges from spending more time on basic lab skills and introductory content to giving students additional activities and going into deeper discussions and content connections. Because groups come in with different levels of experience and prior knowledge, it is difficult to compare the outcomes of the labs from group to group; however, this a strong overall trend of content knowledge gains, increase in career interest, and overall positive experience/association with LabWorks.

\section{DISCUSSION}

Partnerships and Sustainability. The success of CORD's programming would not have been possible without the collaborative partnership of UAB, McWane Science Center and area teachers. In 1998, Discovery Place and Red Mountain Museum merged to create a stimulating interactive science museum now called McWane Science Center, which is the cornerstone of Birmingham, Alabama's downtown revitalization. Every year McWane welcomes hundreds of thousands of visitors ranging in age from ages 0-100 and from states across the South. Year-to-year, McWane is consistently voted as an Alabama family favorite and has placed in lists of top 10 children's science museums in the US. McWane has remained dedicated to the partnership with UAB CORD since the beginning of the GENEius and BioTeach programs. They continue to give dedicated space for two lab classrooms, staff workspace and the free use of conference rooms. They also have a reservation department that handles bookings and museum admission billings (for non-grantfunded groups), an on-site cafe and lunchroom, bus parking, and additional activities for students while on a field trip. Note, CORD does not charge the students or schools any fees for the experiences in GENEius or LabWorks. In the summer, McWane continues to give full use of the labs to CORD for teacher training programs and provides workshop participants with free parking. All these facility and logistic supports are vital to assisting schools in planning and managing field trips, and these resources lend themselves to an effective synergistic partnership (Dunn et al., 2020). In return, GENEius and LabWorks provide middle and high school programs that are very important in fulfilling McWane's mission to advance science understanding of all students. In $2016 \mathrm{McWane}$ recorded 82,881 students as having participated in field and group trips. Most of these participants were in grades preK-5, which is the museum's main audience. However, the mission and goals of the museum extend to learners of all ages. By collaborating with UAB CORD, McWane can continue to offer high-quality programming for middle and high school students without investing their staff's time and resources. There are only a 
few similar full-time programs in other science museums that have a strong bonding of the resources of a Research 1 university and an outstanding children's science museum. That bonding provides outstanding UAB STEM students to facilitate these programs at McWane, and for the facilitators the program affords great teaching experiences that will inform their communication ability in their future STEM careers.

The programs also benefit the mission of McWane, since much of the equipment and reagents required for CORD labs would be very expensive for the museum's typical operating budget and the labs' low student to teacher ratio would be difficult to make possible with museum education staff alone. Most importantly, McWane or any other museum would have a difficult time financially supporting the necessary STEM expertise that is provided to the GENEius and LabWorks programs by CORD staff and UAB faculty and trainees. While McWane has had to add on additional charges for its programming to survive rising costs, CORD has been able to maintain stable pricing for the museum entrance fees and together with McWane, grants from Alabama Power and other businesses, and other grant and contracts, thus facilitating ensuring that no one who wants to come to our labs is turned away. Thus, McWane benefits from an overall increase in revenue at almost no direct cost to the museum, and through these programs, the museum can offer much more high-level secondary programming than would be available otherwise.

As vital as the sustained partnership with McWane Science Center has been for the longevity and success of CORD, many other partnerships have made the sustainability of CORD possible. Another group of critical players has been the local school districts and classroom teachers. It is important to include school stakeholders in partner programs like CORD's (Myende, 2019), since without teacher support and partnership, the program would be much more challenging. In BioTeach and its middle school corollary GeoTeach, the teachers actively modify our current laboratory experiences and create new experiences, which the teachers present at the end of the course as their final project. The best new/modified projects have been incorporated into CORD's offerings at McWane and in school classrooms. Thus, as they return to their classrooms, the teachers feel ownership in the projects. We also query the teachers about the experiences they and their students have at each CORD offering, thus providing critical feedback, which the teachers recognize that we incorporate it into future offerings. We have worked directly with district and state school board members, district administrators, school administrators, and teachers to build and sustain trusting relationships. Often, high needs schools are very cautious of inquiry-based STEM education, for fear that students will not be easily managed. By providing the experiences at LabWorks and GENEius, the teachers gain experience and skills in hands-on teaching and can come to a better understanding of how hands-on experience can greatly facilitate STEM learning. They can then effectively advocate for such experiences in their classrooms.

The need for STEM teacher professional development has changed greatly over the past two decades. Twenty years ago, there were relatively few options for professional development for secondary teachers, few high-level science laboratories. By contrast, in recent years such offerings are almost overwhelming to teachers relative to both the options offered and demands on teacher preparation time. Teachers have come to trust CORD's offering of high-quality, standards-connected professional development and a welltrained STEM professional staff, who assist the teachers all year around. This has kept CORD connected with its teachers and has ensured their participation in CORD programs. We also strive to ensure that all programs, whether for teachers or students, are responsive to current needs in general and relate to the current concerns of students. A vital part of a successful university-school partnership is a balance of understanding goals and needs of the school district and addressing these with programming in which the university has expertise (Thorkildsen and Stein, 1996). CORD regularly takes feedback from teachers, who have attended labs and professional development as well as from administrators, and then we use this data to refine labs and develop new offerings.

Also, over the last 20 years, we have seen changes within area schools that have made sending students on field trips more challenging. As the demands on school budgets increase, bussing has proven more difficult for teachers to secure for field trips. Further, liability issues have dissuaded most schools from allowing parents to bring the children from school to outside experiences. Also, the increased dependence on standardized testing makes it more difficult for teachers to secure approval to be out of the classroom for a single or multi-day field trip. While we cannot remove these hurdles, we have been able to help schools find means of funding busses and substitute teachers and have worked with administrators to demonstrate the value of our programming for their students' STEM education. The long-lasting relationships and mutual respect that we have built up with teachers and school systems helps to keep them engaged in our programs (Farah, 2019).

Funding. Sustaining funding of programs on this scale is no small feat. It has required applying to different sources and a willingness to adapt and expand our programs to grow with changing needs in the STEM education world. The BioTeach program was initially funded through UAB and a Howard Hughes Medical Institute grant. Later, BioTeach was funded through the Alabama Commission on Higher Education and US Department of Education. Some support has also come 
through Gear Up partnerships with LEAs. Much of the longevity of both GENEius and LabWorks, as well the creation of GeoTeach has been made possible through National Institute of Health SEPA grants, with additional funding through the National Science Foundation. Private funding for laboratory space was provided by Altec-Styslinger Foundation, Inc. and McWane. Including the stipends for the teachers in BioTeach, we estimate that the BioTeach course and the provisioning of classroom modules to the teachers has an annual cost of about $\$ 40,000 /$ year.

GENEius was initially funded as part of the Howard Hughes grants and an early SEPA award. From about 2003, the primary funding was through competitive grants from the Alabama Commission on Higher Education, GEAR UP grants (pass-through from US Department of Education), Alabama Power access grants, the Altec-Styslinger Foundation, McWane, UAB and local donors. It has been important for the continued UAB funding that the programs increase the number of students from underserved schools who enter $\mathrm{UAB}$ and successfully complete STEM education majors. The annual cost of GENEius is about $\$ 70,000$.

LabWorks was initially funded by a UAB-McWane partnership, with some funding from Alabama Power and private donors. In 2005, the program received substantial NIH SEPA funding and has since been funded by subsequent SEPA awards, GEAR Up grants, Alabama Power access grants, McWane, UAB, the City of Birmingham and local donors. The annual cost of GENEius is about $\$ 55,000$.

Overall, one of the most difficult tasks is to continuously fund programs like the ones described here. Most grant mechanisms last for 3-5 years, and at their conclusion, any new grants must demonstrate a new direction in order to gain renewed funding. It is clear from our partnerships with schools that funding to assist with busses and substitute teachers has become very important to allow schools to be able to send students on field trips. This should be considered by anyone interested in offering programming in a format similar to ours.

Our success with the programs described herein, are the result of outstanding collaborators and a willingness of CORD staff to modify or totally revamp our programs in relation to feedback received from teachers, students and parents and in relation to the enormous changes in science and technology that have occurred over the past two decades. Seeking sustained funding is not an easy process, but it is ultimately necessary, and the earlier that such funding is pursued, the more likely it will be that successful programs can be sustained.

Challenges and Barriers. While applying for and maintain funding are inherent challenges of sustaining a grant-funded project, we would like to share information about some of the specific challenges we have encountered in the hopes that it helps similar programs avoid them.

Funding for partner schools and field trips was an essential part of the initial SEPA funding for LabWorks. Teachers from partner Title-I schools were able have their substitute teacher cost and bus fees reimbursed by the projects. Also, the science center has secured private funding to aid admission cost for students from these schools, which has also helped to maintain their attendance. However, when the initial LabWorks funding ended in 2010-2011, we saw a sharp decline in attendance as many Title I schools had relied on those funds to make transportation possible (see Figure 3). It has been our experience that it is easy for schools to bring cafeteria lunches so funding meals is not essential, but obtaining funds for substitute teachers, transportation, and program fees is very important to ensure that all teachers and students who want to participate are able to attend.

Accommodating larger class sizes has also been a challenge, particularly for the LabWorks program. Most teachers request to bring all of their students and often an entire grade level. In a standard departmentalized public middle school, this can easily be over 100 students per teacher and 100-400 students per grade level. When the GENEius and LabWorks spaces run the same program simultaneously, we can seat approximately 55 students per lab. Because LabWorks programs are only two hours, the lab can be done twice daily and we can accommodate about 110 students per day. We believe a low student to teacher ratio is important for the success of the labs due to considerations like safety when using dissection equipment or chemicals, instruction on novel equipment and techniques such as micro pipetting, and asking probing questions and letting students share ideas during open inquiry.

As teachers and principals find it harder to take field trips (largely due to constraints from standardized testing and funding), they must make difficult choices about their outings. For many schools, although they would like to bring students to the lab, they can only take field trips which will accommodate all students in a grade level in a single day and address specific STEM learning standards.

Although it is the goal of these programs to improve STEM education in Alabama, we understand that a single day or experience is not enough to close the opportunity gap facing many of our students. We therefore have multiple avenues to try to extend experiences for the student and teacher. All lab programs are aligned to state standards so that teachers can use them to enhance content they are teaching, whether it be as an engaging introduction, summative experience, or review later in the semester. We have had successes in creating partnerships with individual teachers in which they bring their students to the lab multiple times throughout the year so that students can build on skills developed in the first lab. However, as we discussed previously, there seems to be more difficultly in teachers being able to secure busses 
and permission for field trips. Therefore, we have connected these school-year programs with extended summer opportunities. Our summer camps are housed at the University as the science center uses most of their space for their own camps. However, the GENEius lab space stays reserved for BioTeach and GeoTeach, which is advantageous to the science center, since it allows us to highlight to the teachers that the museum is a great field trip destination for their students.

Lessons Learned. It is critical for teachers, school administrators, community members, and higher education to partner in the education enterprise to prepare students for the STEM careers of the 21st century. Each partner must realize their role and respect the special knowledge and skills that other partners bring to the table. In CORD's experience, many teachers from all K-12 levels shy away from teaching unfamiliar science content and even experienced teachers can seem like novices when teaching new content (Sanders et al., 1993). Further, many teachers do not want to try teaching inquiry-based lessons, because they are concerned that their students cannot handle the freedom and that their classroom will dissolve into chaos. Professional development and content support encourage the teachers to implement new inquiry-based science activities in the classroom. Sustained programs such as BioTeach, GeoTeach, GENEius, and LabWorks help to meet the demand for student engagement in "real science" and give teachers multi-modal exposure to high-level content and inquiry in action. The teachers not only see and experience cutting-edge science being well taught, but they also realize that classroom management can be maintained. Often, teachers report using the experiences in GENEius and LabWorks to convince their administrators that hands-on science can work effectively without threatening classroom decorum.

Over the last 20 years, CORD has learned important lessons that identify best practices and guide CORD's future programs, including the following:

Importance of Strong Relationships with Local Education Agencies. The importance of building and maintaining a strong, trusting relationship with stakeholders from local school districts cannot be overstated. There is a history of mistrust by teachers and administrators relative to higher education partnerships. This is particularly seen in schools in urban settings, which often feel that higher education sees the school systems as a method to bring in funding to the university/college, and thus, schools often see higher education placing a great amount of work on them, while they receive little in return. Also, schools often feel that partnerships are ways for universities to tell schools how to improve their education process (Fitzgerald, 2008; Tröhler, 2017). CORD has worked with administrators at all levels and individual teachers to build relationships of trust, in which the teach- ers understand that they are valued and respected partners. CORD approaches these relationships from attitude of collaboration. We strive to know what the schools and teachers believe is needed, so that what we offer is truly supportive.

We also encourage teachers to bring all students to lab programs. Often, teachers only want to bring their best-behaved students. We want to make all students feel welcomed, even if they exhibit behavior problems (for which we train our staff in methods to handle misbehavior). Often the kids who are suspect or initially disruptive are the ones who are most engaged by hands-on science.

We have always included teachers and administrators in the conversation about what they would like or need to come out of new grant programs for which we apply. We base our K-12 grants on work with focus groups of teachers and administrators, always querying them about what area of STEM is most important, what areas of science are most difficult to convey to students and how we can best facilitate their teaching efforts. We always start with the notion that we will learn as much from the teachers as they learn from us. Also, we typically include one to three administrators as co-Investigators on CORD grants, providing adequate resources to make them true partners.

The importance of this dialogue and partnership is never felt more keenly than when we have not had it. While there have been some stalwart supporters among our LEAs for over two decades, there has also been a high rate of turnover. New administrators, especially in districts that have many "failing" schools, are eager to close their doors to all outside initiatives, for fear that those programs would detract from their aggressive attempts to improve education. This gives them an opportunity to regroup the schools that they are leading, but it removes beneficial programs and support from many teachers and students. Some methods we have found to be effective in working through these situations include having many contacts in a district, so that a change in personnel does not entirely close the door on our relationship. We also treat changes in leadership and policy as opportunities to recognize and provide space to the schools to regroup, while we stay close on the sidelines, looking for opportunities to reengage. Typically, administrators will return to the table once they have had time to settle into their new role.

Additionally, it is important to communicate training initiatives with administrators. If they see the value of their students attending a field trip to the GENEius lab, they are much more likely to approve the request. Moreover, if they are supportive of teachers engaging in inquiry-based or $5 \mathrm{E}$ science (Bybee, 1997), teachers are much more likely to implement new methods they learned in the professional development; methods that they might otherwise have concerns about trying. 
Effective Teacher Training Program Design. Appropriate compensation and adequate recognition are important to engage teachers, but it is equally important to provide a program that relates closely to their assigned course of studies and can be implemented at their schools. Relative to compensation, we have found that payments to teachers of $\$ 100$ / day for extended summer training is reasonable. The teachers can either take this as pay or pay for the tuition for the UAB graduate course associated with the program. About $30 \%$ of the BioTeach participants take the course for graduate credit, while most take it for district continue education credits. During the school year, we provide a smaller stipend for workshops or offer free workshops with the cost of substitute teachers reimbursed. Both are effective in recruiting teachers.

In CORD programs, teachers are required to attend $100 \%$ of the training to get paid for their attendance (unless a documented extenuating circumstance is provided). Using this mechanism, we typically obtain $95 \%$ attendance at all summer BioTeach and GeoTeach sessions (including those for the summer of 2020, which went virtual due to the COVID pandemic). It has also been important to determine the necessary contact hours needed to provide teachers with adequate training. In recent years we have observed teachers having greater demands on their summer schedules as state courses of study have changed, districts have adopted new initiatives and more professional development programs have become available and are often required by districts. Any successful, program must be seen by teachers as worth their time. In addition to providing stipends, materials, and training, some additional incentives provide opportunities to meet and collaboratively learn from university faculty and to receive continued support from CORD program directors and the faculty throughout the year. It is important for the CORD staff and faculty involved to see the process as a collaborative one in which they learn from the teachers as the teachers learn from them.

Importance of Standards-Based Training. Teachers are faced with a great pressure to show exactly where their lesson plan fits into the state or national standards. We have found greater implementation of our programs and more fidelity to them when participants know exactly where the modules they have trained on fit into the standards. We strive to develop modules that integrate into multiple standards, deviate from the "textbook" examples and connect content to meaningful STEM stories and careers.

Strong Linkage to Research-Mentors. A lack of adequate knowledge and skills can leave a teacher shying away from inquiry-based lessons for which he/she is not prepared. thus it is important that CORD directors are excellent in their STEM knowledge, respect the teachers, and professional de- velopment and content support that encourages the teachers to implement new inquiry-based science activities (Birman et al., 2000). The CORD staff are available year-round to assist the teachers when they run into any difficulties. The CORD facilitators and faculty understand that the K-12 and college classroom must be transformed to meet the challenges of the 21 st century. Our teacher groups and UAB facilitators have understood that in the classroom, the "didactic only" format of the 20th century will not prepare their students for dynamic 21 st century STEM-related careers, and thus they are transforming their teaching and the teaching of those under them to a more interactive, inquiry-based format.

Need for Relatable Role Models. There is a need for role models, to whom the students can easily relate, especially for students in communities that are historically underrepresented in biomedical research and other STEM careers. STEM education that lacks these role models will struggle to prepare diverse students for 21st century careers. Through aggressive recruitment and CORD's partnership with the UAB Vice President for Diversity, Equity and Inclusion, over 50\% of facilitators have been minorities, many of whom grew up in the same school systems we serve. Since CORD staff and facilitators have relatively short-term interaction with students, it is important for the UAB student facilitators to be diverse role models to demonstrate to the diverse students who come to LabWorks and GENEius that they too can become STEM professionals (more than $60 \%$ of students in CORD programs are African-American, and another $20 \%$ are from rural, underserved school systems; CORD facilitators are about $50 \%$ female and $35 \%$ African-American). Studies that evaluate the effectiveness of science programming show student and facilitator relationships greatly promote STEM learning (Fields, 2009).

It is important for higher education, $\mathrm{K}-12$ teachers, school administrators, community partners, and student facilitators to partner in the education and research enterprise, with each realizing and acknowledging the special knowledge and skills that others bring to the table (Bevan et al., 2010). Given an environment of respect and understanding, with a touch of patience on all sides, universities and communities can come together to advance the education of K-20 students, and thereby enhance the learning of all, including the public. For BioTeach, LabWorks and GENEius, partnering with a local science center has benefited the museum and schools and UAB (both in the training that facilitators acquire and by increasing the pathway for area students to eventually enter and succeed at UAB). The safety, high-level laboratory setting, reputation, and physical space of the museum encourages teachers to bring thousands of students to our programs. It is unlikely that this could have been such a robust and sustained program without the active support of all our partnerships. 


\section{AUTHOR INFORMATION \\ Corresponding Author}

J. Michael Wyss.jmwyss@uab.edu

\section{Author Contributions}

The manuscript was written through contributions of all authors. All authors have given approval to the final version of the manuscript.

\section{FUNDING SOURCES}

This study was supported in part by SEPA and Bridges grants from the National Institutes of Health, National Institute of General Medicine (R25 OD016490, R25RR022745, R25 GM111369), the National Science Foundation (DUE 1136327 and DUE 1758292), the Alabama Commission on Higher Education, Gear-Up Birmingham and Gear-Up Jefferson County.

\section{ABBREVIATIONS}

ASIM: Alabama Science in Motion; CORD: Center for Community Outreach Development; ELISA: Enzyme-Linked Immunosorbent Assay; GEAR UP: Gaining Early Awareness and Readiness for Undergraduate Programs; HIV: Human Immunodeficiency Virus; NGSS: Next Generation Science Standards; PCR: Polymerase Chain Reaction; SEPA: Science Education Partnership Award; STEM: science, technology, engineering and math; UAB: University of Alabama at Birmingham; UV: Ultraviolet

\section{REFERENCES}

Bielik, T., and Yarden, A. (2016). Promoting the asking of research questions in a high-school biotechnology inquiry-oriented program. International Journal of STEM Education, 3.

Bevan, B., Dillon, J., Hein, G. E., Macdonald, M., Michalchik, V., Miller, D., Root, D., Rudder-Kilkenny, L., Xanthoudaki, M., and Yoon, S. (2010). Making science matter: Collaborations between informal science education organizations and schools. Washington, DC: Center for Advancement of Informal Science Education.

Birman, B. F., Desimone, L., Porter, A. C., and Garet, M. S. (2000). Designing professional development that works. Educational leadership, 57(8), 28-33.

Bybee, R.W. 1997. Achieving Scientific Literacy. Portsmouth, N.H.: Heinemann.

Chung, Y., Yoo, J., Kim, S.-W., Lee, H., and Zeidler, D. L. (2016). Enhancing students' communication skills in the science classroom through socioscientific Issues. International Journal of Science and Mathematics Education, 14(1), $1-27$. de Vries, L. E., and May, M. (2019). Virtual laboratory simulation in the education of laboratory technicians--motivation and study intensity. Biochemistry and Molecular Biology Education, 47(3), 257-262.

Dunn, M., Drew, C., O’Brien, J., Wood, M., Mora, E., Diener, S., and Perry, D. J. (2020). A community-academic partnership for school-based nonviolence education: The Healthy Power Program. Journal of School Health, 90(1), 65-69.

Ecklund, E.H., James, S.A., and Lincoln, A.E. (2012). How academic biologists and physicists view science outreach. PloS ONE, 7(5), E36240.

Elton-Chalcraft, S., Copping, A., Mills, K., and Todd, I. (2020). Developing research-informed practice in initial teacher education through school-university partnering. Professional Development in Education, 46(1), 5-20.

Farah, Y. N. (2019). Collaborative partnership: opening doors between schools and universities. Gifted Child Today, 42(2), 74-80.

Fields, D. A. (2009). What do students gain from a week at science camp? Youth perception and the design of an immersive research-oriented astronomy camp. International Journal of Science Education, 32 (2), 151-171.

Fitzgerald, T. (2008). The continuing politics of mistrust: performance management and the erosion of professional work. Journal of Educational Administration and History, 40(2), 113-128.

Groark,C., and McCall, R. (2018). Lessons learned from 30 years of a university-community engagement center. Journal of Higher Education Outreach and Engagement, 22(2), 7-30.

Hassell, K.L. (2010). Population estimates of sickle cell disease in the US. American journal of preventive medicine, 38(4), S512-S521.

Jacobi, J. A., Stults, S. E., Shefner, R., Lange, K. E., Abraham, N. S., and Deiger, M. (2019). Connected through content: Chicago teachers partner with Loyola University to build a math and science learning community. Learning Professional, 40(3), 36-39.

Jarrett, K., Williams, M., Horn, S., Radford, D., and Wyss, J.M. (2016). Sickle cell anemia: tracking down a mutation": an interactive learning laboratory that communicates basic principles of genetics and cellular biology. Advances in Physiology Education, 40(1), 110-5.

Myende, P. E. (2019). Creating functional and sustainable school-community partnerships: lessons from three South African cases. Educational Management Administration and Leadership, 47(6), 1001-1019.

Nordqvist, O., and Aronsson, H. (2019). It Is time for a new direction in biotechnology education research. Biochemistry and Molecular Biology Education, 47(2), 189-200.

Sanders, L. R., Borko, H., and Lockard, J. D. (1993). Secondary science teachers' knowledge base when teaching science courses in and out of their area of certification. Journal of Research in Science Teaching, 30(7), 723-736. 
Thorkildsen, R., and Stein, M. R. S. (1996). Fundamental characteristics of successful university-school partnerships. School Community Journal, 6(2), 79-92.

Tröhler, D. (2017). The paradox of being a teacher: Institutionalized relevance and organized mistrust. In: Schools in Transition, 79-95.

Tuysuz, C. (2010). The effect of the virtual laboratory on students' achievement and attitude in chemistry. International Online Journal of Educational Sciences, 2.

Valantine, H.A., and F.S. Collins. (2015). National Institutes of Health addresses the science of diversity. Proceedings of the National Academy of Sciences, 112(40), 12240-12242.

Vawter, D. (2010). Mining the middle school mind. Education Digest, 75(5), 47-49.

Weerts, D. (2019). Resource development and the community engagement professional: building and sustaining engaged institutions. Journal of Higher Education Outreach and Engagement, 23(1), 9-34.

Yang, X., Hartman, M. R., Harrington, K. T., Etson, C. M., Fierman, M. B., Slonim, D. K., and Walt, D. R. (2017). Using next-generation sequencing to explore genetics and race in the high school classroom. CBE - Life Sciences Education, 16(2). 